\title{
An Unconventional Inchworm Actuator Based on PZT/ERFs Control Technology
}

\author{
Guojun Liu, ${ }^{1}$ Yanyan Zhang, ${ }^{1}$ Jianfang Liu, ${ }^{1}$ Jianqiao Li, ${ }^{2}$ Chunxiu Tang, \\ Tengfei Wang, ${ }^{1}$ and Xuhao Yang ${ }^{1}$ \\ ${ }^{1}$ College of Mechanical Science and Engineering, Jilin University, Changchun 130025, China \\ ${ }^{2}$ Key Laboratory of Bionic Engineering, Jilin University, Ministry of Education, Changchun 130025, China \\ Correspondence should be addressed to Jianfang Liu; jfliu@jlu.edu.cn
}

Received 11 July 2015; Revised 27 November 2015; Accepted 28 December 2015

Academic Editor: Hoon Eui Jeong

Copyright (C) 2016 Guojun Liu et al. This is an open access article distributed under the Creative Commons Attribution License, which permits unrestricted use, distribution, and reproduction in any medium, provided the original work is properly cited.

An unconventional inchworm actuator for precision positioning based on piezoelectric (PZT) actuation and electrorheological fluids (ERFs) control technology is presented. The actuator consists of actuation unit (PZT stack pump), fluid control unit (ERFs valve), and execution unit (hydraulic actuator). In view of smaller deformation of PZT stack, a new structure is designed for actuation unit, which integrates the advantages of two modes (namely, diaphragm type and piston type) of the volume changing of pump chamber. In order to improve the static shear yield strength of ERFs, a composite ERFs valve is designed, which adopts the series-parallel plate compound structure. The prototype of the inchworm actuator has been designed and manufactured in the lab. Systematic test results indicate that the displacement resolution of the unconventional inchworm actuator reaches $0.038 \mu \mathrm{m}$, and the maximum driving force and velocity are $42 \mathrm{~N}, 14.8 \mathrm{~mm} / \mathrm{s}$, respectively. The optimal working frequency for the maximum driving velocity is $120 \mathrm{~Hz}$. The complete research and development processes further confirm the feasibility of developing a new type of inchworm actuator with high performance based on PZT actuation and ERFs control technology, which provides a reference for the future development of a new type of actuator.

\section{Introduction}

Precision positioning actuators have been widely researched for applications in various fields such as optics, microrobotics, memory storage, medicine, space, and military [1-3]. Most research efforts for precision actuators have been made on electrostatic $[4,5]$, electromagnetic $[6,7]$, thermal [8-10], and piezoelectric actuation [11]. However, different actuation principles have their own advantages and drawbacks. The electrostatic actuator is typically composed of two electrodes separated by a small gap. Though it has fast response and low power consumption, the sharp nonlinearity of the gapforce relationship limits it to small displacements and makes it not suitable for the applications where displacements are larger than the scale of millimeter. Electromagnetic actuator has many merits such as large displacement, low voltage, and moderate output force, but the complicated electromagnetic structures and difficult fabrication processes restrict its miniaturization. Thermal actuators are found to be more suitable for the fields requiring large force and large displacement under low voltage, but they have slow response. As for the piezoelectric actuators actuated especially by PZT stack materials, they not only can generate large force, extremely precise displacements, but also can have low power consumption and fast response. These characteristics described above make piezoelectric actuation one of the most promising options for developing the advanced precision positioning actuators.

Nature is the best model for an actuator. Inspired by the movement of creatures, various novel actuators based on biomimetic locomotion were developed. For example, Figure 1 indicates the principle of inchworm motion. The inchworm has three pairs of true legs at the front end, like other caterpillars, and only two or three pairs of prolegs at the rear end. An inchworm moves by drawing its hind end forward while holding on with the front legs and then advancing its front section while holding on with the prolegs [12]. 
TABLE 1: Detailed working process of a working cycle of the stepping actuator.

\begin{tabular}{|c|c|c|c|c|c|}
\hline \multirow{2}{*}{ Number } & \multirow{2}{*}{ Valve 1} & \multirow{2}{*}{ Valve 2} & \multicolumn{2}{|c|}{ PZT stack pump } & \multirow{2}{*}{ Hydraulic cylinder } \\
\hline & & & Volume of chamber & Working state & \\
\hline 1 & Close (on) & Open (off) & $V_{0}$ & No working (off) & Stop \\
\hline 2 & Close (on) & Open (off) & $\downarrow$ & Expelling (on) & Move a step \\
\hline 3 & Close (on) & Close (on) & $V_{\min }$ & Stop expelling (on) & Stop \\
\hline 4 & Open (off) & Close (on) & $V_{\min }$ & Stop expelling (on) & Stop \\
\hline 5 & Open (off) & Close (on) & $\uparrow$ & Sucking (off) & Stop \\
\hline 6 & Close (on) & Close (on) & $V_{0}$ & Stop sucking (off) & Stop \\
\hline
\end{tabular}

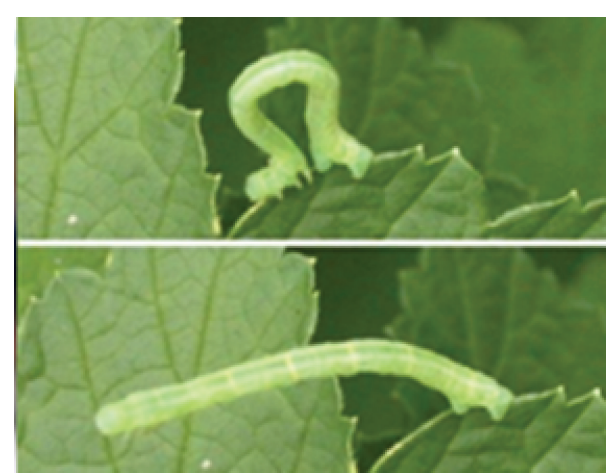

FIGURE 1: The motion photograph of natural inchworm.

Inspired by the inchworm locomotion, Stibitz first reported a novel actuator based on an effective inchworm actuation technology in 1964 and the term "inchworm" was first introduced to describe such devices by Bizzigotti and May in 1975 [13]. Since then, the piezoelectric inchworm actuation technology has drawn significant attention as an alternative of developing the precision positioning actuators to meet future requirements [14-17]. Compared with ultrasonic actuators and piezoelectric inertia friction actuators, the piezoelectric inchworm actuators have shown significant technological advancements in terms of stoke, displacement resolution, driving force, and positioning accuracy.

However, for the conventional inchworm PZT actuator, multiple PZT stacks are required to implement actuation and clamping, which makes the flexible hinge with the amplification function of displacement indispensable. The performance of actuator is highly limited by the machining and assembling accuracy of the clamping mechanism and the variations of the friction coefficient $(0.02-0.15)$ of the contact surface. In addition, the driving load is relatively low in the continuous actuation, and it is possible that the disorders of the clamping position and stepping will occur when the frequency is high [18].

In this paper, a new type of piezoelectric inchworm actuator for precision positioning which differs from conventional inchworm actuation technology is presented. The proposed actuator uses PZT stack pump as the power source, in order to implement the fluid driving with the characteristics of high frequency, high precision, and heavy load. At the same time, it uses the ERFs valve to achieve the switching control of the valve in the high frequency. Finally, through the terminal execution mechanism (precision hydraulic cylinder), it realizes the high precision stepping driving. Different from the traditional PZT inchworm actuator, the most prominent characteristics of the proposed actuator are that the conventional flexible hinge mechanisms which are in charge of mechanical clamp have been replaced by ERFs valves, and precision stepping displacement of execution mechanism is determined by the unit volume change of PZT stack pump chamber. They can improve the coordination of large stroke, high resolution, and large driving capacity for a better effect. Application prospect in the precision positioning field which requires the combination of macro- and microdistance (such as micromachining, precision measurement) can be expected.

\section{Operating Principle}

Since the electrorheological (ER) effect was first identified in the 1940s by Winslow [19], research on ERFs and related engineering devices has been the hot research subject in academic field and attracts attentions of researchers all over the world, and the research progress especially in recent years in ERFs has provided technical support for the development of novel driving mechanism [20-23].

ER fluid is one of the important smart fluids which comprises a suspension of micrometer-sized particles in a dielectric carrier liquid. The electric field stimulus to ERFs will cause a significant increase in the resistance to flow of the fluids [24]. It is just this resistance to flow that results in a pressure drop across the valve, which gives rise to a force to push the motion of the piston. The realization of the ERFs valve function is based on the principle above.

The proposed piezoelectric inchworm actuator utilizes the PZT stack pump to drive the fluids by high frequency and high precision, and it adopts ERFs control technology to realize the high frequency switch control of valves and finally implements the precision stepping actuation with the cooperation of the execution mechanism (precise hydraulic cylinder). Figure 2 illustrates the operating principle of inchworm actuator based on the PZT/ERFs control technology. A complete working cycle of the inchworm actuator can be divided into six steps, the detailed working processes are summarized in Table 1 . Based on the special characteristics of ERFs, when the ERFs valve is charged (switched on), ERFs will transform to the solid phase from the liquid phase immediately, which will result in the closing of the valve, 


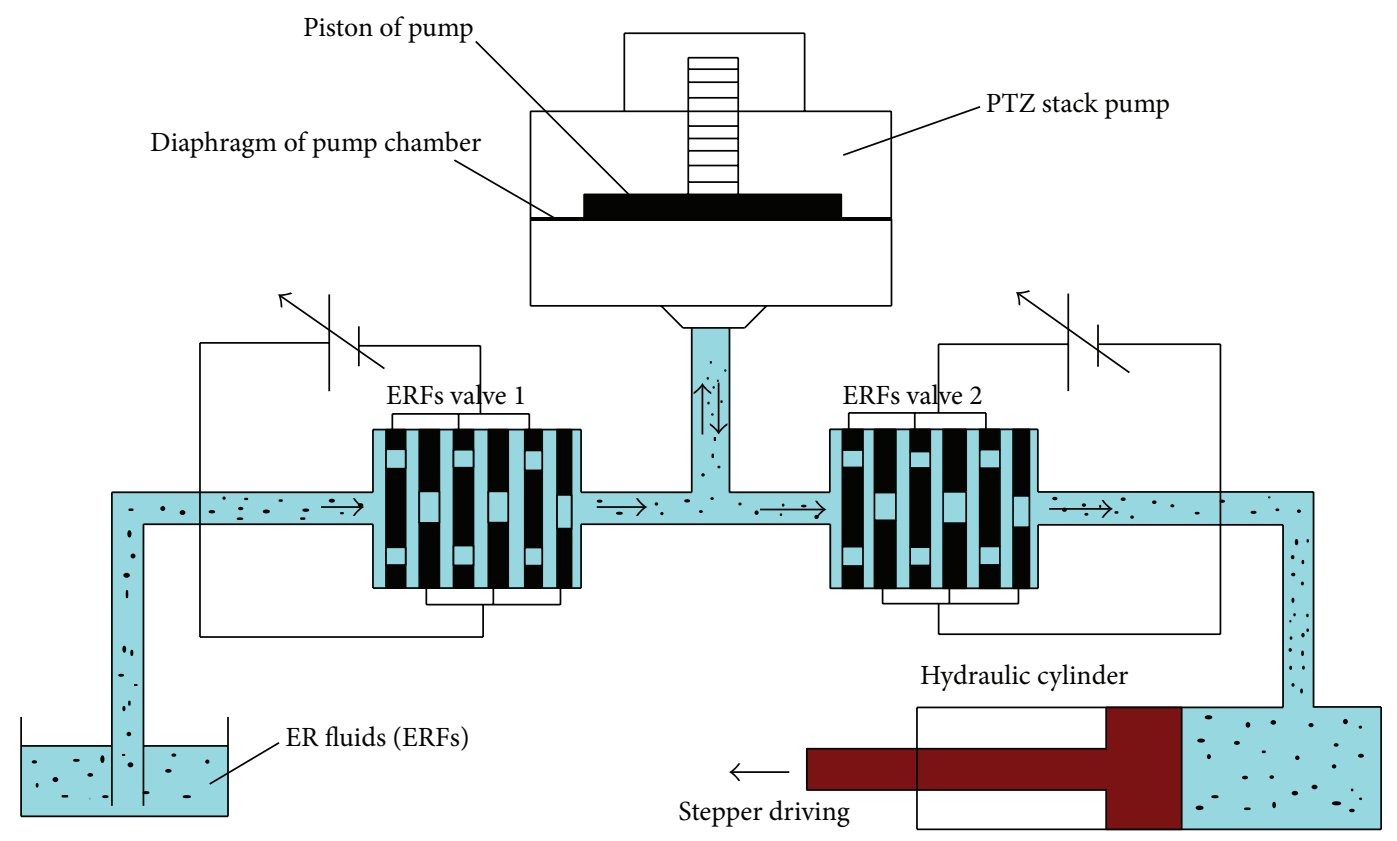

FIGURE 2: The operating principle of inchworm actuator based on the PZT/ERFs control technology.

and if the ERFs valve is switched off, it will remain open. As for the PZT stack pump, when the PZT stack is charged (on) or decharged (off), the PZT stack will be prolonged or retract. Accordingly, it will cause the decrease or the increase of the pump chamber's volume, which will eventually realize the expelling and sucking of ERFs in conjunction with the action of valves. $V_{0}$ and $V_{\min }$ are the initial volume and the minimum volume of pump chamber, respectively. Take the second step in Table 1 as an example. When valve 1 is close and valve 2 is open, the ERFs will be expelled into the precision hydraulic cylinder by the PZT stack pump. Due to the fluid pressure, the ERFs will push the piston and piston rod to one step away in the direction as shown in Figure 2. Through reasonable control of stepping cycle number and frequency, the function of precision positioning will be achieved. In addition, the function of bidirectional actuation for the proposed inchworm actuator can be realized by bridge connection of hydraulic system.

\section{Design of the Inchworm Actuator Based on PZT/ERFs Control Technology}

3.1. Design of PZT Stack Pump. Figure 3 shows the schematic of the PZT stack pump structure. (In order to clarify the working principle of PZT stack pump, the passive check valves are used in Figure 3. However, in actual prototype of actuator, the passive check valves have been cancelled and replaced by the active valves, namely, ERFs valves.) The working principle of PZT stack pump is simple. It utilizes overpressure and underpressure generated by the volume change of pump chamber because of the vibration of seal diaphragm. Also, it uses check valves as "fluidic rectifiers" and finally directs the fluid inside the pump chamber into a desired unidirectional flow. Generally, the mode of volume

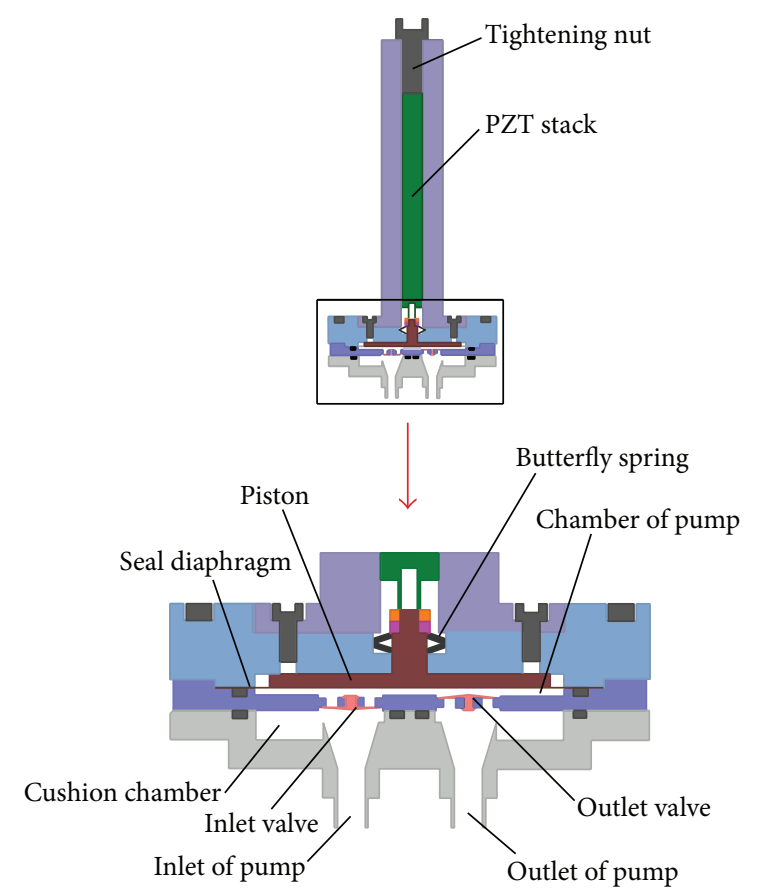

FIGURE 3: The schematic of PZT stack pump structure (with passive check valve).

change of pump chamber can be divided into two categories: diaphragm type and piston type. For the former, the structure is simple and easy to manufacture, but the volume change is small, and the output pressure is directly affected by the diaphragm characteristics. Therefore, under the occasions which require large flow rate and high pressure, the former structure is not suitable for PZT stack pump. For the latter one, the piston type, the volume change of the pump chamber 


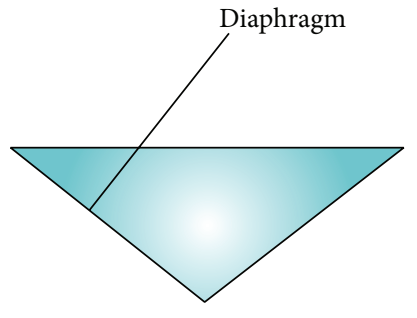

(a) Diaphragm type

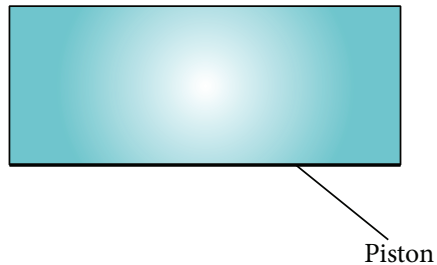

(b) Piston type

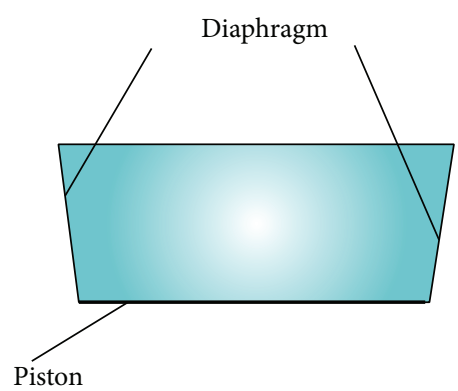

(c) Combined type of diaphragm and piston

FIgURE 4: The schematic diagrams of volume change of PZT stack pump chamber.

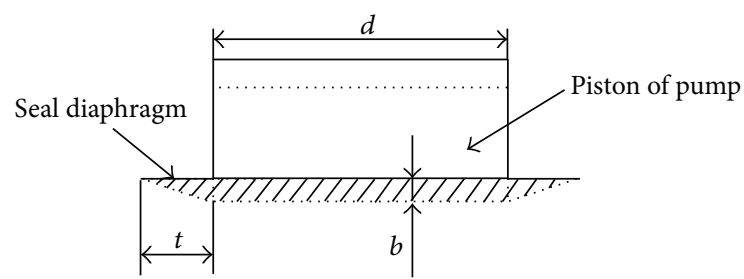

Figure 5: The schematic of the volume variation of pump chamber.

can be controlled more freely, and the output pressure is higher. However, the sealing structure is complex, and the cost of the pump is high. Furthermore, under the condition of high frequency vibration, the working surfaces of piston and cavity wear seriously, which affects the working capacity of pump directly. Integrating the characteristics of the above two structures, a new composite structure is presented in this paper. Figure 4 shows the schematic diagrams of volume change of three structures with the same cavity diameter and output displacement of the PZT stack, respectively.

In view of smaller deformation of the PZT stack, in order to increase the volume variation of pump chamber, a large diameter piston (diameter is $48 \mathrm{~mm}$ ) is designed, to which a metallic seal diaphragm (thickness is $0.7 \mathrm{~mm}$ ) is bonded. Applied by the external excitation, the PZT stack pushes the piston and drives the diaphragm to move up and down, which gives rise to the volume change of pump chamber.

During the design process of PZT stack micropump, the prediction and evaluation of flow rate is a primary task. Figure 5 illustrates the volume variation of pump chamber, which is given by

$$
\Delta V \approx \frac{\pi b}{2}\left[\frac{d^{2}}{2}+t^{2}+d t\right],
$$

where $b$ is the moving distance of piston along stack axial direction, $t$ is the radius difference between piston and seal diaphragm, and $d$ is the diameter of piston. The dotted line represents the position of deformed seal diaphragm pushed by piston, and the shadow part in schematic represents the volume variation. The diameter of piston $d$ and the radius difference $t$ are designed as $48 \mathrm{~mm}$ and $2.4 \mathrm{~mm}$, respectively.

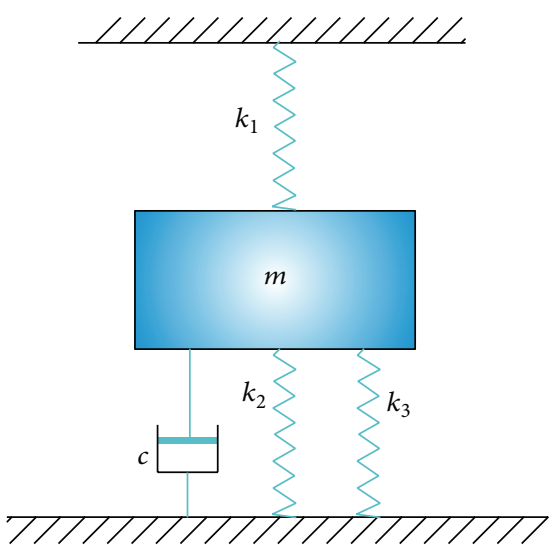

FIGURE 6: The schematic of the vibration model of PZT stack pump system.

When the maximum output distance of PZT stack is $0.1 \mathrm{~mm}$, the ideal flow rate can reach about $98.6 \mathrm{~mL} / \mathrm{min}$ by $15 \mathrm{~Hz}$ working frequency, without considering the system resonance or valve leakage.

In order to maximize the working capacity of the pump, besides ensuring that the deformation of the piezoelectric stack can provide larger volume of changes of pump chamber, it is significantly important that the pump system really works in the resonance state. For the purpose of comprehensive analysis of pump system, the vibration model of system is established, as shown in Figure 6, where $k_{1}$ is the equivalent stiffness of the PZT stack and $k_{2}$ and $k_{3}$ are the rigidity of the seal diaphragm and the butterfly spring, respectively.

Motion differential equations of the system are listed as

$$
\begin{aligned}
m \ddot{x}+c \dot{x}+k x & =F(t), \\
k & =k_{1}+k_{2}+k_{3},
\end{aligned}
$$

where $m$ is mass of system, $k$ is the equivalent stiffness of system, and $F(t)$ is the excitation function of system. When excitation is the sine wave signal,

$$
F(t)=F_{0} \sin \omega t
$$




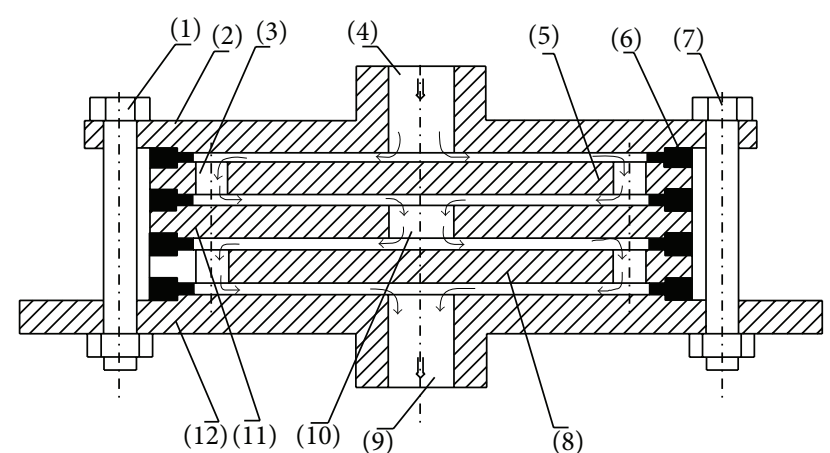
(1) Bolt
(7) Bolt
(2) Upper cover
(8) Electrode number 2
(3) Small orifice channel
(9) Outlet
(4) Inlet
(10) Large orifice channel
(5) Electrode plate number 1
(11) Electrode number 3
(6) Sealing ring
(12) Lower cover

FIGURE 7: The schematic of the ERFs valve adopting the series-parallel plate structure.

where $F_{0}$ is the amplitude of excitation force. Neglecting the intermediate deduction process, the solution of equation can be expressed as follows:

$$
x=\frac{F_{0} / k}{2 \varsigma} \sin (\omega t-\varphi) .
$$

The amplification factor is described as

$$
\beta=\frac{1}{2 \varsigma}=\frac{\sqrt{k m}}{c},
$$

where the relative damping factor is $\varsigma=c / c_{c}$ and $c_{c}$ is the critical damping coefficient. In addition, $c_{c}=2 m \omega_{n}=2 \sqrt{\mathrm{km}}$, where $\omega_{n}$ is the natural frequency of the system.

In the qualitative analysis of the above models, it will be found that the amplification factor of the system is related to the equivalent stiffness, equivalent mass, equivalent damping factor, and so on.

3.2. Design of ERFs Valve. In view of ERFs characteristic of lower static shear yield strength, a composite type ERFs valve is designed, which adopts the series-parallel plate structure. Figure 7 illustrates the structure schematic of the proposed ERFs valve. The positive electrodes (numbers 2, 11, and 12) and the negative electrodes (numbers 5,8 ) are separated by the insulating materials (number 6). The gaps between the electrodes are filled with ERFs and designed to be adjustable. The flow channel through holes (numbers 3,10) is designed on electrodes plates. When switched off, ERFs valve is in open state, and ERFs will easily flow from the inlet (number 4) through the middle channels and holes and finally outflow from the outlet (number 9). Also, when the positive and negative electrodes are applied by electric field, the phase of ERFs transits from liquid state to the quasi-solid state. Then, the valve will perform the closing function. As for the fasteners, the bolts (numbers 1,7 ) not only can play the role of a connection, but also can regulate the gap in a certain range, thereby achieving the desired flow rate.

Based on Bingham model and the relationship between the pressure difference and flow rate in the effect of external electric field, the static equation of fixed plate flow field is established, and the pressure difference between the inlet and outlet without the effect of electric field is given by [21]

$$
\begin{aligned}
\Delta P_{n} & =n \Delta P_{\text {single }}=n\left(\frac{12 \eta_{0} l}{b h^{3}} q+\frac{2 l}{h} \tau_{\mathrm{EY}}\right), \\
l & =R-r_{1}-r_{2}, \\
b & =r_{2},
\end{aligned}
$$

where $R$ is the radius of the electrode plates, $r_{1}$ is the radii of the center holes of electrode plate (numbers 4,9 , and 10), $r_{2}$ is the radius of flow through hole (number 3 ), $h$ is the gap distance between the adjacent electrode plates, $\eta_{0}$ is the viscosity of ERFs without electric field, $n$ is the number of gaps, $q$ is the volume flow, and $\tau_{\mathrm{EY}}$ is the shear yield stress of ERFs. As a result, the pressure difference between the inlet and outlet is subjected to the properties of ERFs, structural dimensions of ERFs valve, and the applied electric field intensity. Based on the principle that the sealing pipe's pressure is equal everywhere, the output force can be calculated by

$$
F_{\text {out }}=\Delta P_{n} \cdot S \text {, }
$$

where $S$ is the size of the area of hydraulic cylinder piston.

3.3. Displacement Resolution of the PZT Inchworm Actuator. Displacement resolution of the inchworm actuator based on the PZT and ERFs control technology is mainly determined by the resolution of flow rate of PZT stack pump and the diameter of hydraulic cylinder piston. Once the design project of precision hydraulic cylinder is determined, the resolution of system will be decided by the flow rate resolution of the PZT stack pump. In order to facilitate the analysis, an assumption is made that the fluids in the system are an incompressible medium without air bubbles. Therefore, if the actual outflow volume of the PZT stack pump in a work cycle is $\Delta V_{i}$ and the inflow volume of the terminal actuator mechanism is $\Delta V_{o}, \Delta V_{i}$ will be equal to $\Delta V_{o}$. Assuming that the actuator in the working process is considered as an approximate static system, the output displacement resolution of its terminal mechanism will be

$$
x_{S}=\frac{\Delta V_{o}}{S}=\frac{\Delta V_{o}}{\pi r^{2}}=\frac{\Delta V_{i}}{\pi r^{2}},
$$

where $r$ is the radius of hydraulic cylinder piston. Taking into account the resonance of the system, the actual volume change $\Delta V_{i}$ is more than $\Delta V$ calculated in (1).

\section{Manufacture of the Inchworm Actuator Based on PZT/ERFs Control Technology}

As mentioned previously, a composite structure has been used in the design of PZT stack pump, which combined 


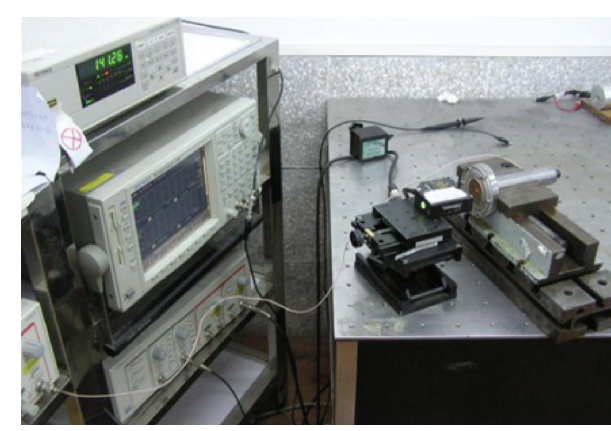

(a)

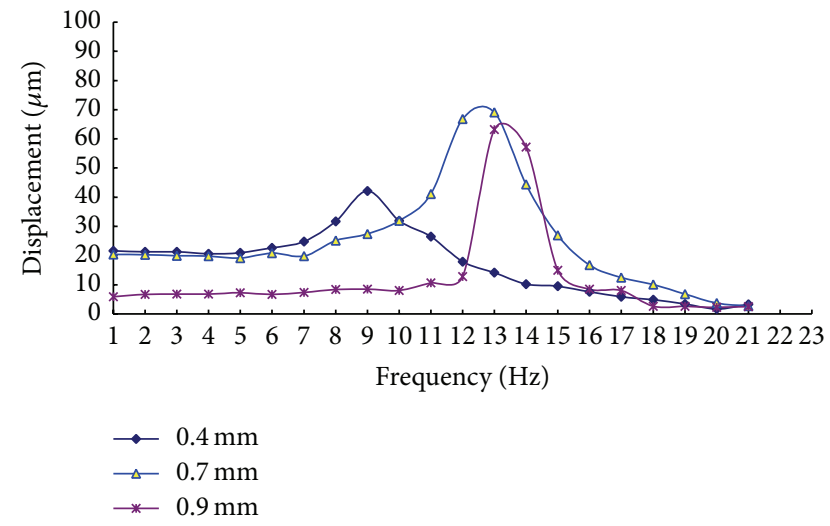

(b)

FIGURE 8: Photograph of the testing system (a) and amplitude frequency characteristic graph of three kinds of metallic seal diaphragm (b).

TABLE 2: PZT stack technology parameters (from Piezomechanik $(\mathrm{PZ})$.

\begin{tabular}{lc}
\hline Model number & PST $150 / 5 / 100$ \\
\hline Length & $105(\mathrm{~mm})$ \\
\hline Nominal displacement & $100(\mu \mathrm{m})$ \\
\hline Maximum output force & $800(\mathrm{~N})$ \\
\hline Rigidity & $8(\mathrm{~N} / \mu \mathrm{m})$ \\
\hline Static capacity & $2.4(\mu \mathrm{F})$ \\
\hline
\end{tabular}

the features of two common types (diaphragm and piston types). Beryllium bronze is selected as the material of metallic diaphragm. In order to study the effect of metallic rigidity on the frequency response characteristics of PZT stack pump, the beryllium bronze sheets of $0.4,0.7$, and $0.9 \mathrm{~mm}$ thickness were selected as the metallic diaphragm. Figure 8 shows the testing picture and testing graph of test system. Analyzing the test data, we can know that, with the increase of the thickness of the metallic diaphragm, the natural frequency of the system is gradually increasing. Among three diaphragms with different thickness, the displacement of the metallic diaphragm of 0.7 thickness is the largest, which accounts for the determination of the optimal thickness of metallic diaphragm. The detailed structure is shown in Figure 3, the diameter of pump piston is determined to be $48 \mathrm{~mm}$, the diaphragm thickness is $0.7 \mathrm{~mm}$, and the diameter of diaphragm is $52.4 \mathrm{~mm}$. The technology parameters of the PZT stack used in pump are listed in Table 2.

Considering the deformation of diaphragm, the noise, and other aspects of working performance of the test prototype, sine wave signal is selected as the working electrical signal. The outflow rate of stack pump prototype tested in the condition of $100 \mathrm{~V}$ voltage and $15 \mathrm{~Hz}$ working frequency reaches $230 \mathrm{~mL} / \mathrm{min}$, which is far higher than the prior prediction performance when not considering the resonance of the system. It is found that the displacement of pump piston has achieved $0.304 \mathrm{~mm}$, and the test result exceeds the nominal displacement of PZT stack. Therefore, it is the system resonance that gives rise to larger improvement of performance of the PZT stack and pump prototype.

In order to achieve a desired working performance of ERFs valve, reasonable structure design is essential besides improving the properties of ERFs. It is an effective design project to appropriately increase the length of valve and reduce the spacing of electrode plate for the improvement of output pressure of ERFs valve, when the electric field intensity and flow rate are determined. A novel series-parallel composite structure is adopted in manufacturing the ERFs valve, which can be decomposed easily and can be changed to increase or decrease the number of intermediate electrode plates according to the actual working situation. During the assembly process, the bolts not only can act on the connection function, but also can adjust the clearance between the electrode plates $(0.6 \mathrm{~mm}-1.4 \mathrm{~mm})$. The test prototype of ERFs valve of four plate series of structure is manufactured in the lab, the maximum output pressure difference of which reaches $290 \mathrm{kPa}$. When the electric field intensity is $3 \mathrm{kV} / \mathrm{mm}$ and the electrode gap is adjusted to $0.8 \mathrm{~mm}$, the output flow rate of valve achieves $100 \mathrm{~mL} / \mathrm{min}$. In addition, with the increase of flow rate, the pressure difference will further increase.

Figure 9 is the photograph of the test project of the inchworm actuator prototype combined with the PZT stack pump and ERFs valves.

\section{Testing and Discussion}

The experimental tests are carried out for the prototype of the new type of inchworm actuator in the lab. Resolution is an important index of performance for the inchworm actuator, which refers to the minimum step size achieved by stepping motion. Figure 10 shows the test results of the displacement resolution. The output displacement of no-load actuator in 20 steps achieves $0.76 \mu \mathrm{m}$, when the driving voltage is $16 \mathrm{~V}$ and the frequency is $1 \mathrm{~Hz}$. Therefore, corresponding to each step, the minimum step size is $0.038 \mu \mathrm{m}$. It is noted that $16 \mathrm{~V}$ 


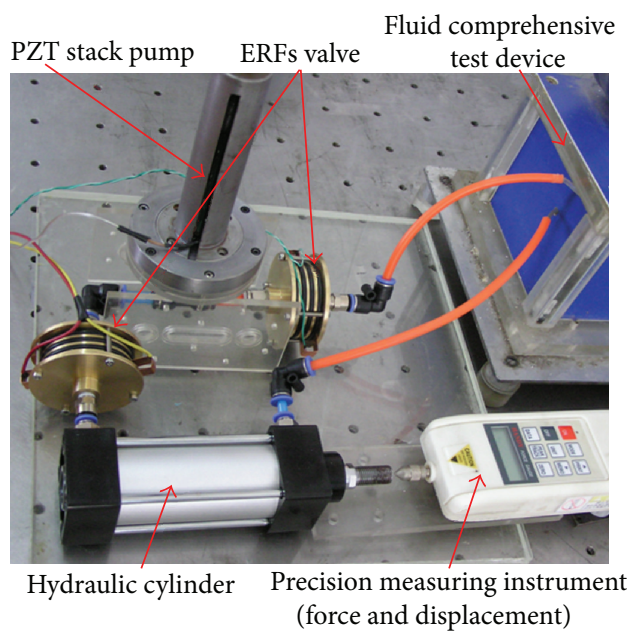

FIGURE 9: The project photograph of the system test.

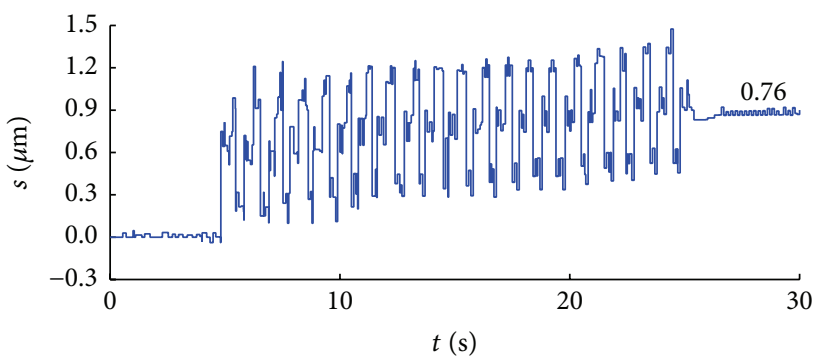

Figure 10: Displacement resolution.

is just the minimum driving voltage for no-load condition. If the system works in load condition, the minimum driving voltage for stable operation will be increased correspondingly. In addition, based on the test graphs, another significant characteristic of actuator was found. Namely, a greater recession happens in each work cycle, and there are some fluctuations at both ends of forward and backward. This test phenomenon is similar to the test result in Li et al.'s research paper [25] about a linear stepper motor based on PZT actuator and ER clampers. In our view, the characteristics of ERFs, the response time of ERFs valve and PZT stack, and the time parameters of control program are the main influencing factors, so if we could improve the characteristic properties of ERFs and optimize the time parameters of electric control program, the recession can be greatly suppressed, and the performance of actuator can be further improved comprehensively.

The static driving force is another important performance parameter of the inchworm actuator. The test results of driving force in different working voltages (for the PZT stack pump) and the same frequency $(1 \mathrm{~Hz})$ are shown in Figure 11, and ERFs valve is applied by electric field of $4 \mathrm{kV} / \mathrm{mm}$. Analyzing the test graph of force versus voltage, it can be found that the output force increases gradually with the increase of voltage. When voltage exceeds $70 \mathrm{~V}$, the maximum driving force achieves $42 \mathrm{~N}$. The value of maximum output force is mainly restricted by the maximum cutoff pressure of ERFs valve. After ERFs valve reaches the maximum cutoff pressure,

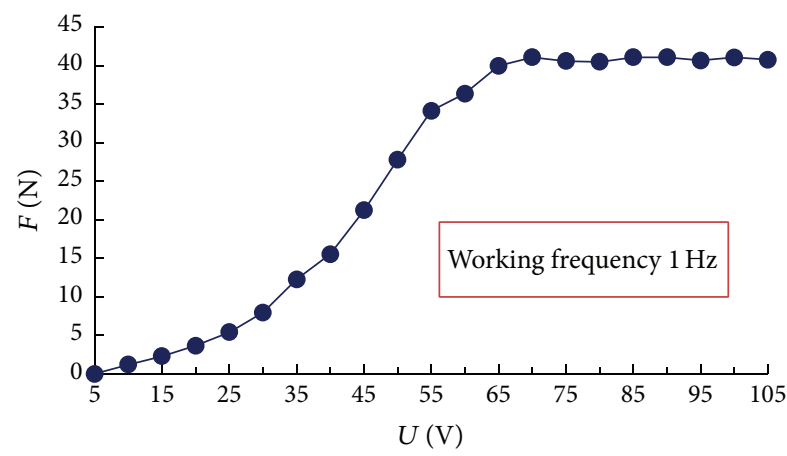

FIGURE 11: Driving force versus working voltage.

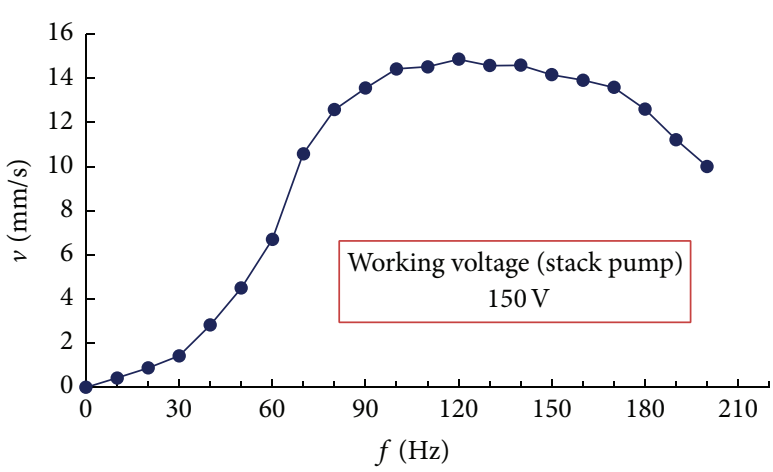

FIGURE 12: Driving velocity versus working frequency.

even if the working voltage of stack pump is increased further, the output load of actuator still cannot increase any more. At this moment, the driving velocity will be improved to some extent. Because ERFs valves replace the complex mechanical clamp structure, the energy consumption in the flexible hinge and clamping mechanism due to deformation and friction has been prevented. With the increase of cutoff pressure of ERFs valves, the output capacity of actuator can be improved further.

The test result shown in Figure 12 indicates the relationship between driving velocity and working frequency of the no-load inchworm actuator, when the PZT stack pump is applied by $150 \mathrm{~V}$ working voltage. Through analyzing the test graph, the optimum driving velocity of actuator system is found. When the working frequency reaches $120 \mathrm{~Hz}$, the maximum driving velocity is $14.8 \mathrm{~mm} / \mathrm{s}$. In addition, within the scope of $30 \mathrm{~Hz}$, there exists an approximate linear relationship between velocity and frequency. When frequency is higher than $120 \mathrm{~Hz}$, with the increase of frequency, the driving velocity reduces gradually. The main possible reasons include the viscosity of ERFs, the internal friction force of ERFs, and adhesion force to the inner wall in the actuator system, which directly give rise to the response lag of ERFs valve behind that of PZT stack pump.

Figure 13 shows the test results about the relationship between the driving force and working frequency for the testing prototype. The driving force of the system shows the declining trend on the whole, with the increase of working frequency. When the frequency is lower than $10 \mathrm{~Hz}$, the 


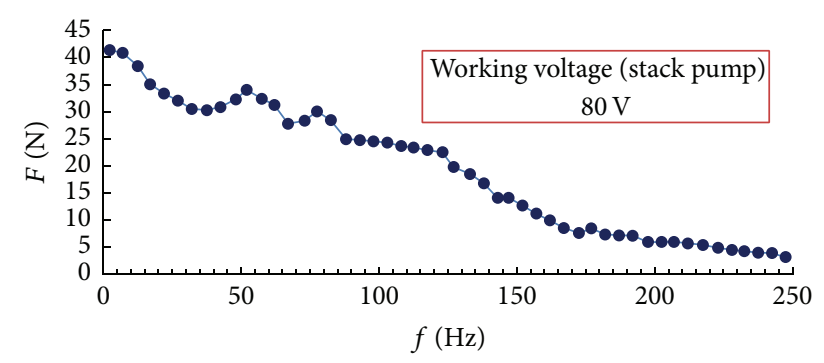

FIGURE 13: Driving force versus working frequency.

driving force remains a relatively stable value (40-42 N). When the frequency is higher than $120 \mathrm{~Hz}$, the driving force shows a significant drop with the increase of frequency. The characteristics stated above are subjected to many factors, such as the internal friction force of ERFs, adhesion force to channel, energy consumption of eddy current generated in the pump chamber, and response delay between fluids and mechanical vibration.

\section{Conclusion}

An unconventional inchworm actuator based on PZT/ERFs control technology is proposed, designed, manufactured, and tested. Different from conventional PZT inchworm actuator, the proposed actuator uses the ERFs control technology in the research and development process. The conventional complex clamping mechanism is replaced by ERFs valve, which can be operated and adjusted easily. The conventional displacement amplification mechanism, namely, the flexible hinge, also has been replaced by the composite diaphragm structure pushed by large diameter piston. The energy consumption in the flexible hinge and clamping mechanism due to deformation and friction has been avoided effectively.

The systematic test shows that the method using PZT actuation and ERFs control technology to develop a new type of inchworm stepping actuator is feasible, and the prototype has demonstrated that it owns the relatively high working performance. The displacement resolution of the unconventional inchworm actuator reaches $0.038 \mu \mathrm{m}$, and the maximum driving force and velocity are $42 \mathrm{~N}, 14.8 \mathrm{~mm} / \mathrm{s}$, respectively. The optimal working frequency for the maximum driving velocity is $120 \mathrm{~Hz}$.

However, as a new actuator using novel actuation technology, the research results still have some limitations. For example, in a signal period, there still exist obvious displacement fluctuation and a greater recession. In order to optimize the working performance of actuator, improving the properties of ERFs and optimizing the control parameters of PZT stack and ERFs valve will be an important research direction in the future.

\section{Conflict of Interests}

The authors declare that there is no conflict of interests regarding the publication of this paper.

\section{Acknowledgments}

This research is supported and funded by the National Natural Science Foundation Projects no. 50605027, no. 51375207, and no. 51475198. The authors thank the professors and faculty members in Key Laboratory of Bionic Engineering (Jilin University) for providing a lot of valuable technical support and help especially in the experiment and test of system prototype.

\section{References}

[1] C.-H. Oh, J.-H. Choi, H.-J. Nam, J.-U. Bu, and S.-H. Kim, “Ultracompact, zero-power magnetic latching piezoelectric inchworm motor with integrated position sensor," Sensors and Actuators A: Physical, vol. 158, no. 2, pp. 306-312, 2010.

[2] M. J. Daneman, N. C. Tien, O. Solgaard, A. P. Pisano, K. Y. Lau, and R. S. Muller, "Linear microvibromotor for positioning optical components," Journal of Microelectromechanical Systems, vol. 5, no. 3, pp. 159-165, 1996.

[3] E. Thielicke and E. Obermeier, "Microactuators and their technologies," Mechatronics, vol. 10, no. 4-5, pp. 431-455, 2000.

[4] M. A. Erismis, H. P. Neves, R. Puers, and C. Van Hoof, "A low-voltage large-displacement large-force inchworm actuator," Journal of Microelectromechanical Systems, vol. 17, no. 6, pp. 1294-1301, 2008.

[5] M. A. Erismis, H. P. Neves, R. Puers, and C. Van Hoof, "Sawtooth vernier ratchets for electrostatic inchworm," Sensors and Actuators A, vol. 156, pp. 66-71, 2009.

[6] H. S. Tzou, H.-J. Lee, and S. M. Arnold, "Smart materials, precision sensors/actuators, smart structures, and structronic systems," Mechanics of Advanced Materials and Structures, vol. 11, no. 4-5, pp. 367-393, 2004.

[7] R. Toda and E.-H. Yang, "A normally latched, large-stroke, inchworm microactuator," Journal of Micromechanics and Microengineering, vol. 17, no. 8, pp. 1715-1720, 2007.

[8] W. Wang, S. Tatic-Lucic, W. L. Brown, and R. Vinci, "Design of a bidirectional MEMS actuator with high displacement resolution, large driving force and power-free latching," Microelectronic Engineering, vol. 85, no. 3, pp. 587-598, 2008.

[9] W. Wang, S. Tatic-Lucic, W. Brown, J. Iceman, S. Hyun, and R. Vinci, "Precision in-package positioning with a thermal inchworm," Sensors and Actuators A: Physical, vol. 142, no. 1, pp. 316-321, 2008.

[10] H. N. Kwon, S. H. Jeong, S. K. Lee, and J. H. Lee, "Design and characterization of a micromachined inchworm motor with thermoelastic linkage actuators," Sensors and Actuators A: Physical, vol. 103, no. 1-2, pp. 143-149, 2003.

[11] H. Lu, J. Zhu, Z. Lin, and Y. Guo, "An inchworm mobile robot using electromagnetic linear actuator," Mechatronics, vol. 19, no. 7, pp. 1116-1125, 2009.

[12] S. Guo, L. Shi, N. Xiao, and K. Asaka, "A biomimetic underwater microrobot with multifunctional locomotion," Robotics and Autonomous Systems, vol. 60, no. 12, pp. 1472-1483, 2012.

[13] S. W. Lee, K.-G. Ahn, and J. Ni, "Development of a piezoelectric multi-axis stage based on stick-and-clamping actuation technology," Smart Materials and Structures, vol. 16, no. 6, pp. 23542367, 2007.

[14] T. Galante, J. Frank, J. Bernard, W. Chen, G. A. Lesieutre, and G. H. Koopmann, "Design, modeling, and performance of a high force piezoelectric inchworm motor," Journal of Intelligent 
Material Systems and Structures, vol. 10, no. 12, pp. 962-972, 1999.

[15] C. Moon, S. Lee, and J. K. Chung, "A new fast inchworm type actuator with the robust I/Q heterodyne interferometer feedback," Mechatronics, vol. 16, no. 2, pp. 105-110, 2006.

[16] J. Kim and J.-H. Lee, "Self-moving cell linear motor using piezoelectric stack actuators," Smart Materials and Structures, vol. 14, no. 5, pp. 934-940, 2005.

[17] T. Hemsel and J. Wallaschek, "Survey of the present state of the art of piezoelectric linear motors," Ultrasonics, vol. 38, no. 1, pp. 37-40, 2000.

[18] J. Li, H. Zhao, X. Qu et al., "Development of a compact 2-DOF precision piezoelectric positioning platform based on inchworm principle," Sensors and Actuators A: Physical, vol. 222, pp. 87-95, 2015.

[19] W. M. Winslow, "Induced fibration of suspensions," Journal of Applied Physics, vol. 20, no. 12, pp. 1137-1140, 1949.

[20] H. Hashizume and H. Shinno, "A study on nanometer positioning table system equipped with electrorheological fluid units," JSME International Journal Series C: Mechanical Systems, Machine Elements and Manufacturing, vol. 43, no. 1, pp. 183-189, 2000.

[21] S.-M. Chen and C.-G. Wei, "Experimental study of the rheological behavior of electrorheological fluids," Smart Materials and Structures, vol. 15, no. 2, pp. 371-377, 2006.

[22] Y. Kondoh and S. Yokota, "Actuators making use of electrorheological fluids: movable electrode type ER actuators," Journal of Intelligent Material Systems and Structures, vol. 10, no. 9, pp. 718-722, 1999.

[23] K. Yoshida, K. Kamiyama, J.-W. Kim, and S. Yokota, "An intelligent microactuator robust against disturbance using electrorheological fluid," Sensors and Actuators A: Physical, vol. 175, pp. 101-107, 2012.

[24] R. Stanway, "Smart fluids: current and future developments," Materials Science and Technology, vol. 20, no. 8, pp. 931-939, 2004.

[25] C. Li, Y. Meng, and Y. Tian, "Recession in a linear stepper motor based on piezoelectric actuator and electrorheological clampers," Smart Materials and Structures, vol. 21, no. 12, Article ID 125008, 2012. 


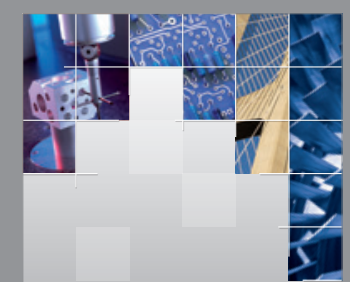

\section{Enfincering}
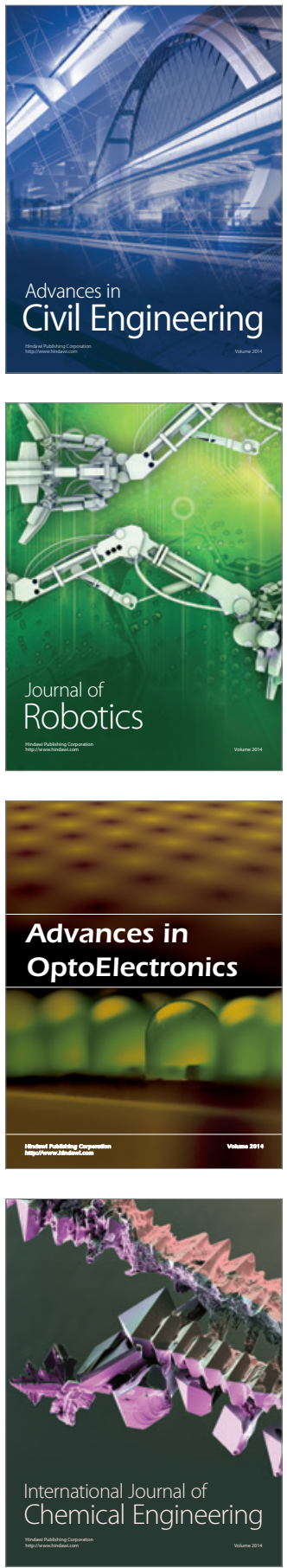

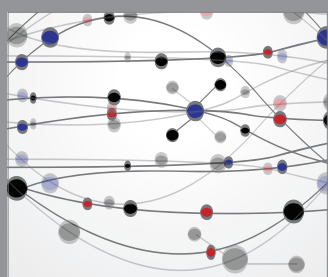

The Scientific World Journal

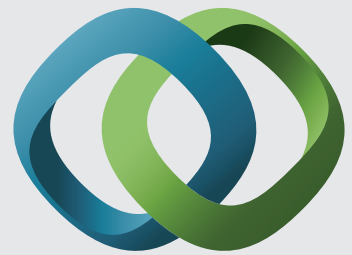

\section{Hindawi}

Submit your manuscripts at

http://www.hindawi.com
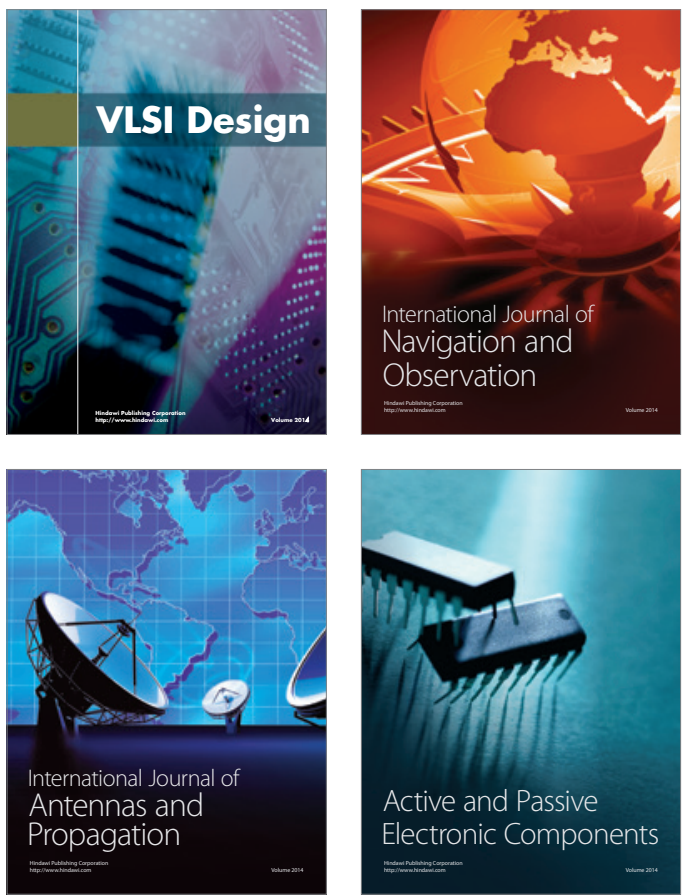
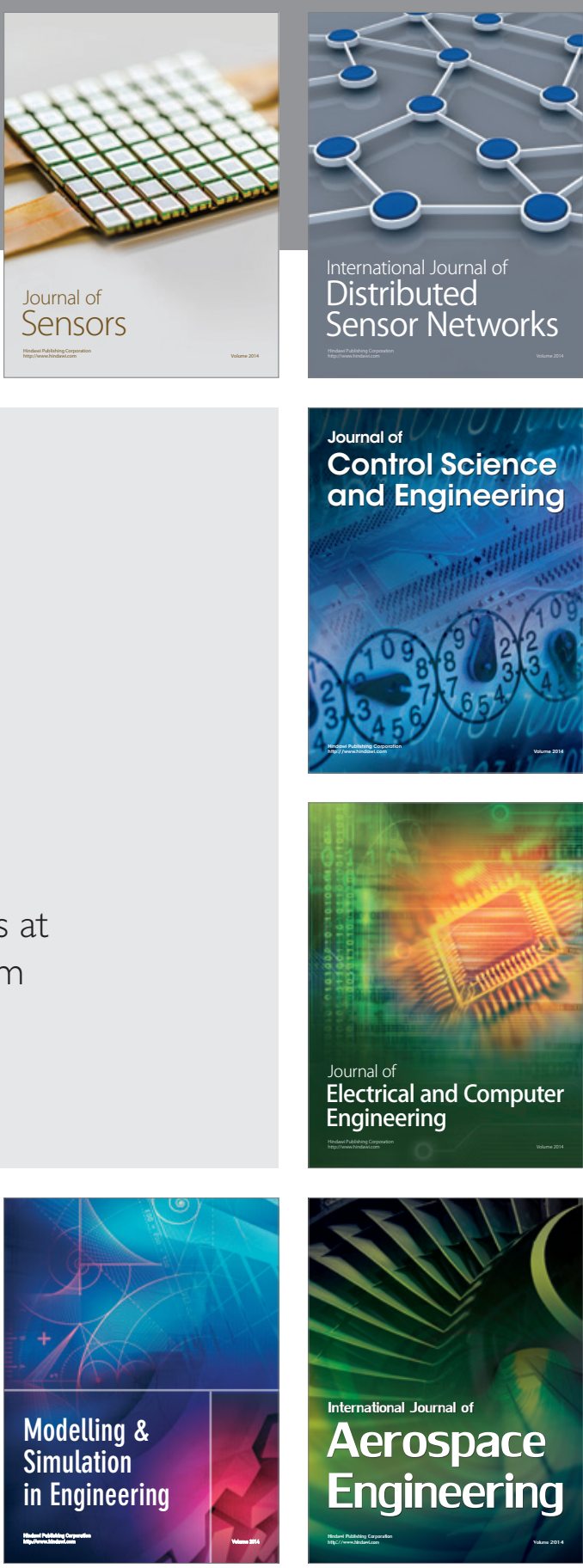

International Journal of

Distributed

Sensor Networks

Journal of

Control Science

and Engineering
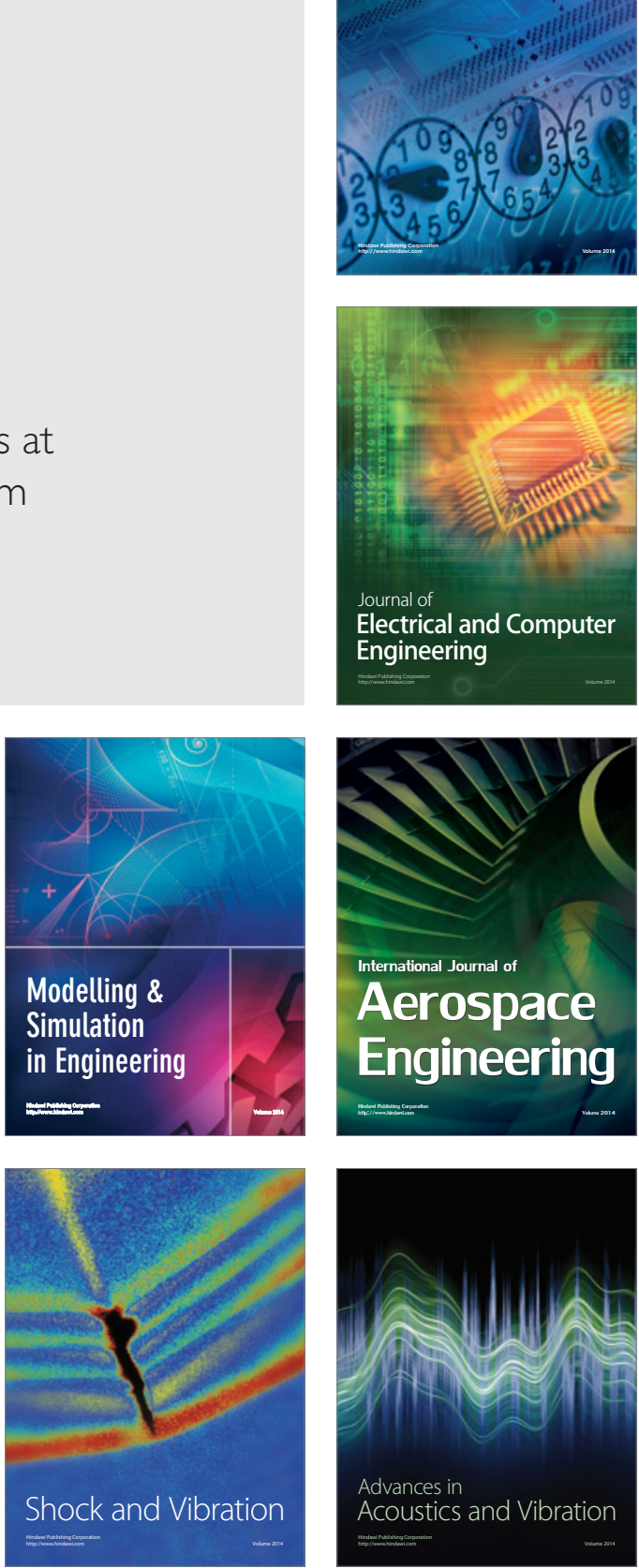